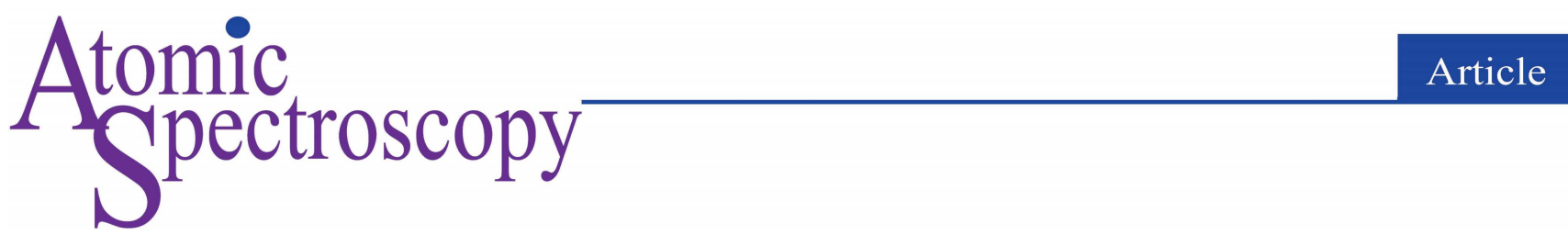

\title{
In Situ Micro-XRD Methods for Identifying Glass and Minerals in Extraterrestrial Samples
}

\author{
Jiaxin Xi, ${ }^{\text {a,b,c }}$ Lingya Ma, ${ }^{\text {a,b,* }}$ Haiyang Xian, ${ }^{\text {a,b }}$ Gaofeng Wang, ${ }^{\text {a,b }}$ Jieqi Xing, ${ }^{\text {a,b,c }}$ Jingming Wei, ${ }^{\text {a,b,c }}$ Jianxi Zhu, ${ }^{\text {a,b,c }}$ \\ and Hongping $\mathrm{He}^{\mathrm{a}, \mathrm{b}, \mathrm{c}}$ \\ ${ }^{a}$ CAS Key Laboratory of Mineralogy and Metallogeny/Guangdong Provincial Key Laboratory of Mineral Physics and Materials, Guangzhou Institute of \\ Geochemistry, Chinese Academy of Sciences (CAS), Guangzhou 510640, P.R. China \\ ${ }^{\mathrm{b}}$ CAS Center for Excellence in Deep Earth Science, Guangzhou 510640, P.R. China \\ ${ }^{c}$ University of Chinese Academy of Sciences, Beijing 100049, P.R. China
}

Received: December 01, 2021; Revised: February 04, 2022; Accepted: February 04, 2022; Available online: February 07, 2022.

DOI: $10.46770 / A S .2022 .016$

ABSTRACT: Glass or amorphous state materials are vital components of lunar regolith and have attracted considerable attention. The analytical data on the texture and structure of glassy and crystallized materials can be used to reconstruct the geological history of the Moon. However, it is often challenging to distinguish glass from crystals based on morphology and elemental composition, especially in complex extraterrestrial samples that have been subjected to significant impact and metamorphism. In this study, micro-X-ray diffraction ( $\mu \mathrm{XRD}$ ) techniques were used to identify crystalline minerals and glassy phases in lunar breccia samples extracted in the Chang'e-5 mission. The samples were processed using different operating methods to identify a technique that would minimize damage to their original appearance and structure and yield optimal results. The diffraction results, which possessed two-dimensional patterns were classified into dominant dispersion halos, concentric diffraction rings, independent diffraction spots, and coexisting rings and spots. The results correspond to four types of sample states, namely, the amorphous material, polycrystal, single crystal, and a mixture of polycrystals and single crystals. By identifying the crystallization state and phases of various samples in a non-destructive and intuitive manner, $\mu \mathrm{XRD}$ can facilitate in situ analysis of special samples generated during important geological events, thereby promoting the understanding of complex origins and evolution of extraterrestrial bodies.

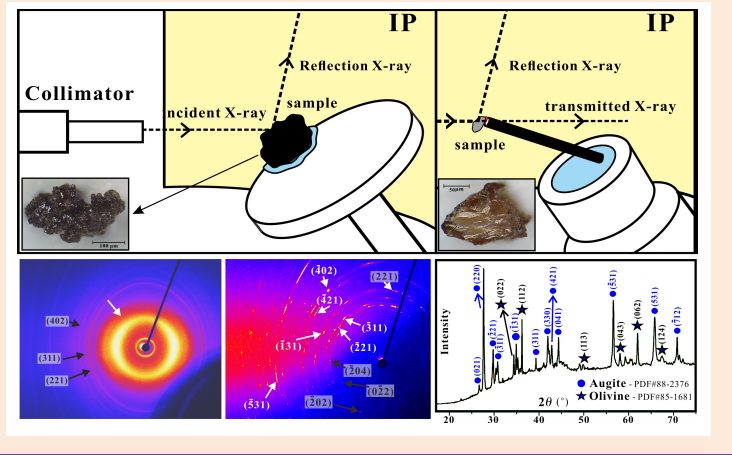

\section{INTRODUCTION}

Lunar regolith contains abundant fragments of mare basalt, breccia, and glasses (20-30 vol\%). ${ }^{1-4}$ Lunar glassy materials generated mainly by volcanic eruptions and impact processes on the Moon can help clarify the geochemistry of the lunar interior, impact melting processes associated with the lunar crust, and impact the chronological history of the Moon. ${ }^{3-5}$ Glasses that do not have schlieren and exotic mineral/lithic inclusions are commonly regarded as pristine glass. ${ }^{5}$ Breccias with a vitric matrix can be categorized as soil and light matrix breccias, which differ in the color, content, and origins of glass components. ${ }^{6-10}$ According to research on Apollo samples, light matrix breccias are not always of pyroclastic origin and are likely to be products of meteoroid impacts. ${ }^{10-12}$ Micrometeorite impact generates glass and agglutinates from crystallized minerals, leading to complex coexistence patterns of glass and microcrystals. ${ }^{11-13}$ These patterns can provide valuable information regarding the impact history (such as the impact position and pressure) and thermal history and considerably influence the spectral characteristics of the solid surface of the Moon. ${ }^{14-18}$ Research on the texture and structure of glassy materials and crystals in a particular geological environment is expected to facilitate the analysis of the original composition and impact metamorphism history of local rocks. However, it is challenging to identify and study lunar glassy materials in the laboratory and remote sensing data because of 
their complex form, origin, and composition. Several studies on Apollo samples have identified the occurrence of glasses and these glassy and crystallized materials have been distinguished according to their morphology and elemental composition using techniques such as scanning electron microscopy (SEM), energy dispersive X-ray spectrometry, and Raman spectroscopy. ${ }^{10-14}$ However, minerals coexist and mingle with glassy phases in extraterrestrial samples that have with broken lattices that have undergone impact metamorphism and melting. This renders the in-situ investigation of glass components with microcrystals challenging.

$\mathrm{X}$-ray diffraction (XRD) is a commonly used and effective tool for analyzing the structure of materials ranging from powders to solids and fluids. It can also be used to characterize the threedimensional atomic structures of crystalline materials. ${ }^{14}$ Some related techniques have been widely used for material identification and characterization. ${ }^{19-22}$ Notably, micro-XRD $(\mu \mathrm{XRD})$ and XRD patterns were used to investigate the phase composition and structural characteristics of the Moon surface. ${ }^{10-}$ ${ }^{14}$ Several reports have used XRD techniques to analyze the main mineral composition of the relevant materials to understand the geological characteristics of the Moon. ${ }^{14-18,23}$ For example, Taylor et al. performed quantitative XRD (QXRD) analyses on Apollo soil samples with different compositions and ages, using a modified CheMin XRD instrument to determine the bulk mineral abundances. ${ }^{14}$ It was demonstrated that powder-based XRD could identify the main mineral phases of an aggregation bulk. However, this approach is less effective for identifying microcrystals, crystallites, and skeletal crystals of the glass components of specific particles. ${ }^{14}$ Moreover, the relationship between the crystals and coexisting glass remains extensively examined. To this end, it is necessary to perform structural analysis with a high spatial resolution and flexible sample preparation technique. As the conservation of sample properties is being prioritized over research, studies involving destructive analytical methods such as powder XRD and single-crystal XRD are expected to be limited. ${ }^{19}$ In situ $\mu \mathrm{XRD}$ is a non-destructive technique that does not significantly degrade or alter the appearance and internal structure of the sample. This versatile technique uses a two-dimensional (2D) general area diffraction detector system to acquire information regarding texture and crystallinity and discriminates multiple phases through the characteristic structural parameters of minerals. ${ }^{21,22}$ In a $2 \mathrm{D} \mu \mathrm{XRD}$ pattern, a single crystal produces discrete spots, whereas a polycrystalline sample produces circular arcs, that is, Debye (Debye-Scherrer) rings. Spots and arcs correspond to certain families of lattice planes defined by the Miller index $(h k l) .{ }^{24-26}$ Moreover, dispersed rings are generated from coherent scattering by noncrystalline substances.

Hence, the objective of this study is to demonstrate the efficiency of multiple $\mu$ XRD methods to identify glass and microcrystals in a sample and crystallite and skeletal crystal about the glassy material. Furthermore, we discuss the geological factors that influence the structural characteristics of materials in lunar samples extracted during the Chang'e-5 mission. Experiments were performed to obtain fine XRD patterns of samples in an intuitive and non-destructive manner. In addition, these experiments also provided a reference for selecting the test conditions and preparation method for $\mu$ XRD analysis of unique samples such as extraterrestrial rocks. The findings suggest that the diffraction patterns of samples can help explain the impact history of the Moon by defining the phase composition and structure of the sample, thereby demonstrating the potential of related $\mu$-XRD techniques in theoretical research.

\section{EXPERIMENTAL}

Samples. The examined samples were scooped from the lunar regolith surface during the Chang'e-5 mission of China. The Chang'e- 5 spacecraft landed at $43.06^{\circ} \mathrm{N}, 51.92^{\circ} \mathrm{W}$, approximately $170 \mathrm{~km}$ east-northeast of Mons Rümker. ${ }^{27}$ Two lithic clast samples, CE5C0000YJYX023 (60.5 $\mathrm{mg}) \quad$ and CE5C0000YJYX125 (137.3 mg), were obtained from the China National Space Administration. Large particles $(\leq 0.05 \mathrm{~mm})$ were collected from sample vessels. Two types of samples were prepared for the $\mu \mathrm{XRD}$ analysis. For the first type, bare particles were used, and for the second type, the particles were mounted using epoxy resin.

Instrumentation and Operating Conditions. In situ $\mu \mathrm{XRD}$ patterns were recorded using a Rigaku D/MAX RAPID-V diffractometer (Tokyo, Japan) with a microfocus rotating anode source (MicroMax-007HFM X-ray generator) and a curved imaging plate (IP) detector (Fig. 1a-b). The diffractometer was operated with $\mathrm{Cu}-\mathrm{K} \alpha$ radiation $(\lambda=1.5418 \AA)$ generated at $40 \mathrm{kV}$ and $30 \mathrm{~mA}$. The $\mu$ XRD patterns were collected using a $0.03 \mathrm{~mm}$ collimator for $1200 \mathrm{~s}$ to ensure adequate intensity.

Epoxy mounts with particles were attached to a circular sample stage with a slightly smaller diameter than the size of the epoxy mounts used here and rotated with a fixed $\omega$-axis $\left(21^{\circ}\right)$ of the sample stage, and $\phi$ fluctuating from $-30^{\circ}$ to $120^{\circ}\left(1^{\circ}\right.$ per second $)$ to produce the maximum beam footprint of the sample. For the breccia clasts, clusters were pasted on the sample stage using a tiny amount of Vaseline ${ }^{\circledR}$ with the smallest possible contact area (Fig. 2a). Several other particles that exhibited flaky shapes were attached to one end of an insulated capillary (Fig. 2c). In general, both frameworks can be used to record $\mu$ XRD patterns of any sample that can be mounted on the sample stage without colliding with the beam optics (Fig. 2a and 2c). Before the diffraction experiments, the visual field and focus were manually adjusted to 

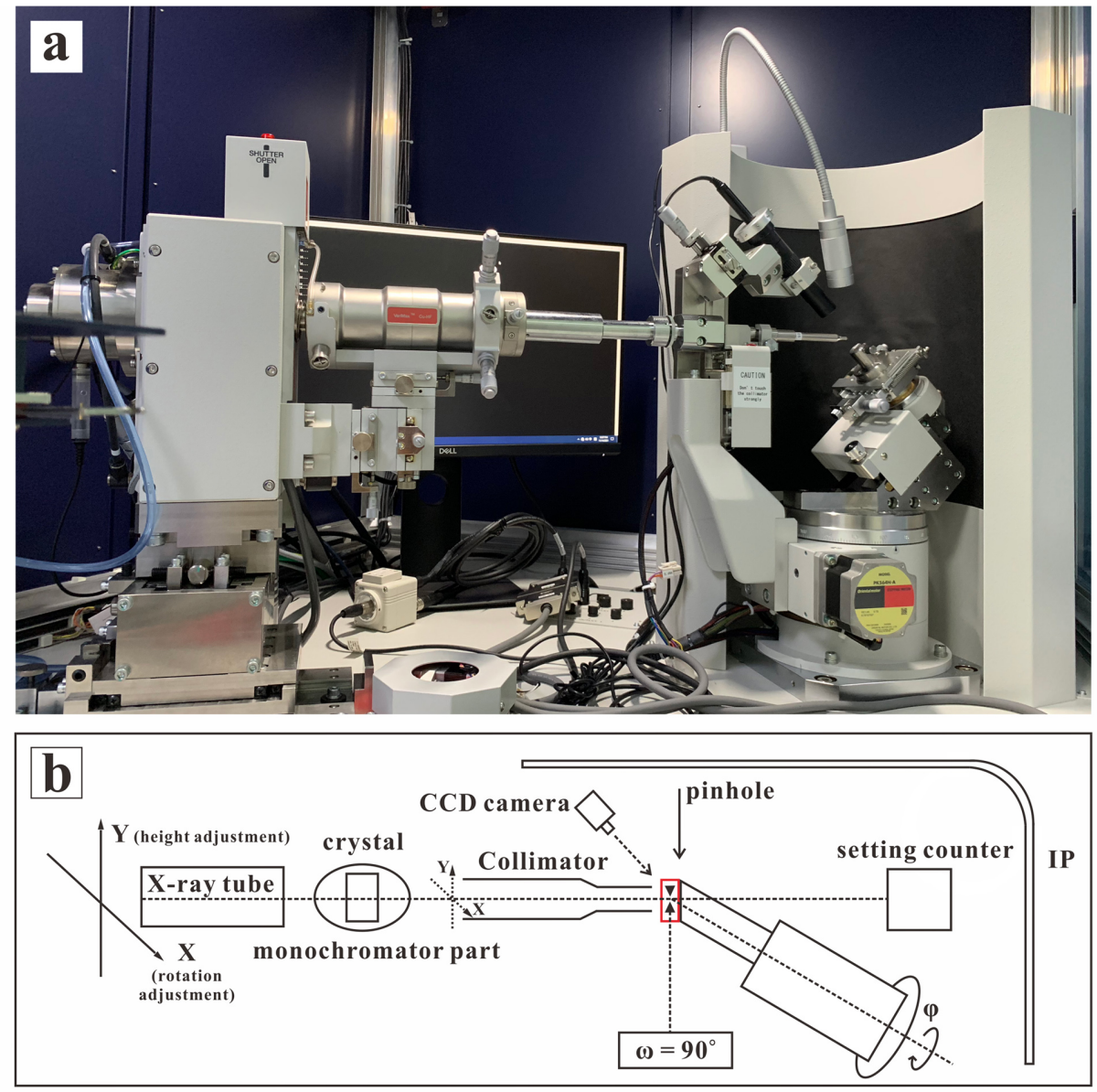

Fig. 1 (a) Photograph and (b) structural diagram of the Rigaku D/MAX RAPID-V diffractometer.
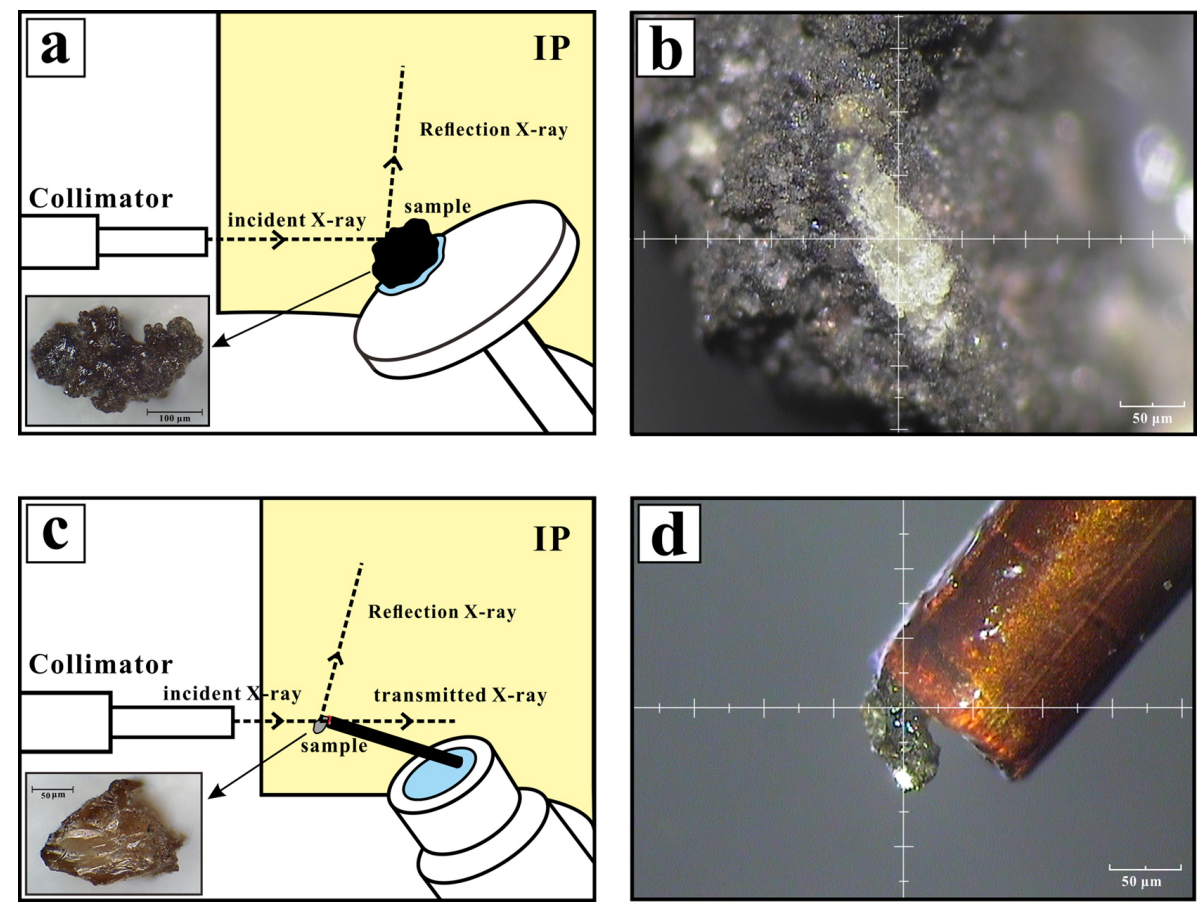

Fig. 2 Schematic of two sample holding methods for $\mu$ XRD analysis based on the Rigaku D/MAX RAPID-V diffractometer. Diagram of the beam path, sample stage, fragment sample cluster, and IP detector (a) when the sample is attached directly to the stage and (c) when a capillary holds the sample. (b) and (d) show images corresponding to (a) and (c), respectively, obtained using the optical microscope of the Rigaku D/MAX RAPID-V diffractometer. 
Fig. 3 Occurrence and imaging principle of Debye rings. When the incident X-ray satisfies the Bragg equation of a certain crystal plane, the generated diffracted lines form a conical diffraction surface: the path of the incident $\mathrm{X}$-ray is the axis, the irradiated sample area corresponds to the vertex, and the vertex angle is $4 \theta$. Each crystal plane with a Miller index ( $h k l)$ diffracts and produces a specific conical surface. Concentric rings form on the negative film and can be characterized.
Fig. 4 Typical 2D diffraction patterns of Luna breccia clast samples obtained using the method shown in Fig. 2a. The diffraction rings and spots are matched with the corresponding Miller indices (marked by arrows). A typical dispersion halo is marked by the white arrow in (a). In (b), the Xray diffraction signals involve two phases: the primary and secondary phases are marked by white and black arrows, respectively. observe the sample clearly, and the target of the sample was placed in the center of rotation of the sample stage with an optical microscope mounted on a Rigaku D/MAX RAPID-V diffractometer (Fig. 2b and 2d).

The data were collected as 2D images containing Debye rings, corresponding to the intersection of a cone of diffracted rays with the Ewald sphere. ${ }^{15,20-21}$ Generally, each crystal plane group that satisfies the Bragg equation forms its reflection cones and leaves Debye rings on the negative film when a monochromatic X-ray irradiates polycrystalline samples (Fig. 3). Therefore, the intensity distribution along the Debye rings can be analyzed to identify the phase and microstructure. The diffracted X-ray intensity was converted to intensity profiles versus $2 \theta$ using the Rigaku 2DP software. For each XRD pattern, the interpretable $2 \theta$ region was manually selected.

\section{RESULTS AND DISCUSSION}

Bare particles of breccia clasts. Two methods that can yield diffraction patterns in 2D images were used to hold the samples on the stage for the $\mu$ XRD analysis (Fig. 2a and 2c). The diffraction spots and Debye rings provide useful information regarding the concentration and granularity of the crystallite phases under analysis. After eliminating the interference of the binder (Vaseline), the $\mu$ XRD results of the lunar breccia clasts obtained using the first method can be divided into three typical patterns, namely A-1, A2, and A-3 (Fig. 4a-c). In A-1 (Fig. 4a), the diffraction pattern appears as a thick, diffuse, and irregular ring with several thinner rings and contains no diffraction spots. The typical diffraction halo is attributable to the abundance of glass (marked by the white arrow in Fig. 4a) and electron coherent scattering in amorphous materials that lack long-range order. ${ }^{28}$ Moreover, a few Debye rings (marked by the black arrow in Fig. 4a) can be observed, corresponding to a poorly crystallized augite (PDF\#41-1483) polycrystal. In A-2 (Fig. 4b), the continuous and irregular concentric rings indicate that the sample was polycrystalline in the form of fine particles, as suggested by the thin arc. ${ }^{14,28}$ Lattice planes diffracted by pyroxene-type and less albite-type minerals can be observed on most Debye rings. The arrows in Fig. $4 \mathrm{~b}$ represent the locations, at which the one-dimensional (1D) integration diffraction pattern (intensity vs. $2 \theta$ ) was obtained (A-2 in Fig. 5), corresponding to augite (PDF\#24-0203) and anorthite (PDF\#78-2330). In A-3 (Fig. 4c), sharp and independent diffraction dots with tiny connections and extremely thin lines appear in the diffraction pattern. These dots indicate the presence of a single crystal with a coarse particle size (beyond the scale range of the beam spot), identified as crystalline Na-rich anorthite (PDF\#20-0528). Significant differences between the three diffraction patterns can also be observed in the 1D spectra converted from the 2D images (Fig. 5). Notably, sample A-1 
Fig. 5 1D spectra ( $2 \theta$ versus intensity) of Luna breccia clast samples converted from two-dimensional images. One-dimensional spectra of A-1 (black line), A-2 (red line), and A-3 (blue line) are converted from the twodimensional images shown in Figs. 4 (a), (b), and (c), respectively.

Fig. 6 Typical 2D diffraction patterns and converted one-dimensional spectra ( $2 \theta$ versus intensity) of Luna breccia clast samples, obtained using the method shown in Fig. 2c. (a, b) 2D patterns of samples obtained using the method shown in Fig. 2c. The diffraction rings and spots are matched with the Miller indices of the corresponding diffracted crystal planes (marked by arrows). (c) 1D spectra of B-1 (black line) and B-2 (red line) converted from the two-dimensional images shown in (a) and (b), respectively.

yielded a broad hump owing to X-ray scattering, especially in the $2 \theta$ range of $15-25^{\circ}$. Samples A-2 and A-3 exhibited similar background values, and their phase retrieval results are shown in Fig. 5, with the matched reflection planes of each mineral crystal.

All complanate samples held by a capillary in the $\mu \mathrm{XRD}$ analysis exhibited the coexistence of glass and crystals (Fig. 6a-b)
Fig. 7 Lunar breccia samples with epoxy mounts and diffraction patterns of different parts of the samples. (a) SEM images of lunar samples with epoxy mounts. (b-e) diffraction patterns of different parts of the samples. The crystal planes are matched with the corresponding diffraction rings or spots. In (c) and (d), XRD signals are identified as two phases: the primary and secondary phases are marked by white and black arrows, respectively.

Samples composed of glass and anorthite polycrystals (Fig. 6a, B-1; PDF\#85-1660) and those exhibiting the coexistence of glass, single crystal, and pyroxene polycrystals (Fig. 6b, white arrows in B-2; PDF\#86-0004) with a small amount of ilmenite (Fig. 6b, black arrows in B-2; PDF\#73-1256) were identified. When the patterns were converted to 1D spectra (Fig. 6c), the background intensity was related to the proportion of glass in the sample, and the X-ray fluorescence effect was significant. The X-ray fluorescence effect is generally simulated by $\beta$ lines that correspond to the excitation of the inner electrons and outer electrons filling the vacancies, during which a secondary characteristic X-ray is emitted. ${ }^{29}$ The occurrence of this effect is indicated by the irregular extending diffuse red halo in Fig. 7c-e. Mineral phases were identified by comparing the observed diffraction patterns with the PDF-2 database.

Epoxy-mounted samples. The diffraction results of samples with epoxy mounts (Fig. 7a) have a distinct characteristic: the diffracted signal of the low-angle region $\left(2 \theta=0-20^{\circ}\right)$ next to the bright center of a $2 \mathrm{D}$ image is obstructed by the epoxy perimeter (Fig. $7 b-e)$. Figure $7 b-e$ indicates a progressive increase in crystalline 
Fig. $81 D$ spectra ( $2 \theta$ versus intensity) of Lunar breccia samples with epoxy mounts, converted from two-dimensional images. The 1D spectra of C-1 (black line), C-2 (red line), C-3 (blue line), and C-4 (green line) are converted from 2D images shown in Figs. 7(b), (c), (d), and (e), respectively. The results of phase retrieval are indicated on each spectrum, along with the Miller index of the diffracted crystal planes.

material in sample positions from C-1 to C-4. A diffuse halo, diffraction spots, and Debye rings coexist (Fig. 7b), indicating that glass and polycrystals coexist within the irradiation region. The diffraction patterns shown in Fig. $7 \mathrm{c}$ and $7 \mathrm{~d}$ are the most common patterns observed in more than 50 results acquired from the samples. A combination of single and multiple crystal patterns can be observed, with spots, ring patterns, or intermediate discontinuous "spotty rings". Such patterns indicate the presence of a mixture of crystals and crystallites of several sizes and strong orientations. The $h k l$ planes (Fig. 7c) correspond to the characteristic reflections of augite (PDF\#88-2391; Fig. 8c). In contrast, the $h k l$ planes in Fig. $8 \mathrm{~d}$ correspond to augite (PDF\#882376) and olivine (PDF\#85-1681). The diffraction pattern of C-4 (Fig. 7e) exhibits bright, sharp spots with minimal connections, suggesting single crystals of olivine (PDF\#85-1681) with a small amount of pyroxene (PDF\#86-0004) (Fig. 8e). Similar to the diffraction results of the breccia clasts, the background intensity in the converted $1 \mathrm{D}$ spectra is closely related to the content of glassy material in the analyzed samples.

Moreover, the data noise from the cluster samples directly fixed on the sample stage (as described in the previous section) was higher than that from the relatively flat surface of the epoxy mount. However, the intensities of all results were adequate for structural analysis and phase identification. Because the reflection and transmission signals generated by X-rays, were simultaneously received, the surface planeness of the complanate samples held by the capillary did not considerably influence the results.

Clarification of geological events associated with the crystalline state. Early research on the glass and cement components of lunar soil has focused on the influence of lunar surface weathering on the spectral characteristics of the soil..$^{30-37}$ Nevertheless, after the Apollo samples were obtained, research attention to amorphous material gradually shifted to the association of the material characteristics with the hightemperature evaporation deposition on the lunar surface, ${ }^{31-39}$ sputtering deposition of the solar wind, ${ }^{15-18,31,40,41}$ and impact events of meteorites. ${ }^{42,43}$ According to a recent theory, an amorphous membrane is expected to originate from impact. Most research on lunar meteorites and Apollo samples ${ }^{31-33,48}$ mainly composed of breccia ${ }^{44-47}$ and have proven that the Moon experienced frequent and strong impact events. Many impact events significantly changed the properties of minerals on the lunar surface, ${ }^{1-4,49-52}$ and glass distribution became widespread and extensive. ${ }^{10-13,44,53}$ In this study, we collected over 300 XRD data points on the samples with epoxy mounts. However, no pure diffraction dispersion halo belonging to glassy materials was observed in these samples, likely because of the following two aspects. Firstly, during sample preparation, the incorporation of glass-ceramic $(<0.1 \mathrm{~mm})$ was avoided. Secondly, the lunar regolith contains a high percentage of small crystals (such as various mineral fragments, microcrystals, crystallites, and skeletal crystals), which are agglutinated by glass. ${ }^{5,14}$ The presence of glasses suggests that a thick, glassy cover was present on the grain boundary, and the results simultaneously exhibited the diffraction characteristics of glass and minerals. The reference to such a glassy cover has been derived in other studies and is considered an essential characteristic of evaporation deposition ${ }^{30-32}$ or melting ${ }^{31,33-38}$ caused by high-energy meteorite impact.

According to the 1D spectra (Figs. 5 and 8), when a broad hump of glassy materials (Figs. $5 \mathrm{~A}-1$ and $8 \mathrm{C}-1$ ) is observed, the diffraction peaks of the crystals tend to be broad and less sharp than those in other spectra (Figs. 5 A-2 and A-3 and 8 C-2, C-3, and C-4), suggesting a lower crystallinity. These spectra with glassy characteristics correspond to $2 \mathrm{D}$ images that exhibit broader streak-like features (Figs. 4a and 7b). These features represent a large number of crystal lattice defects and are indicative of a more disordered crystal structure. According to the experiments, the XRD intensity distribution along the Debye rings changes from small discrete spots in unshocked single crystals to increasingly long arcs as the impact magnitudes increase. ${ }^{19,54}$ This change occurs because impact waves with increasing shock 
pressure break crystals into progressively smaller and increasingly misoriented subdomains, which may destroy the long-range order in the crystal lattice, that is, solid-state amorphization/diaplectic glass formation. ${ }^{19,49,54}$ Crystal subdomain sizes decrease with complex effects such as fracturing, cleaving, slipping, and kinking, leading to the presence of streak-like features in the diffraction patterns of single-crystal materials prior to the shock event. ${ }^{54}$ Therefore, a position/particle with a higher glass content can be inferred to be closer to the impact center or volcano eruption center.

Furthermore, it is generally believed that glasses with soil-like compositions have an impact origin. ${ }^{4,55-56}$ Based on the mineral phases identified in this study and related results reported in previous studies, ${ }^{4}$ the coexistence of single crystals, polycrystals, and glasses corresponds to an impact origin. The glass composition is expected to facilitate the determination of the origin of breccia in future work.

\section{CONCLUSIONS}

The results of the $\mu \mathrm{XRD}$ analysis of multiple lunar samples involved four types of 2D diffraction patterns: (1) a dispersion halo without Debye rings and diffraction spots identified as a glassdominant pattern; (2) Debye rings without any independent spots corresponding to polycrystal dominance; (3) separate diffraction spots with extremely thin connections between them corresponding to single-crystal dominance; (4) the coexistence of a dispersion halo, Debye ring, and diffraction spot, representing the simultaneous occurrence of a single crystal, polycrystalline, and glassy phase, respectively. The widths of the diffraction spots and Debye rings helped clarify the crystal particle size and order of the crystal lattice.

Considering the results observed in this study, we conclude that $\mu \mathrm{XRD}$ is an effective tool for the non-destructive and intuitive in situ analysis of the crystallization state of a sample. Structural information can be obtained from different types of samples subjected to different $\mu$ XRD-based methods, which were selected according to the morphological characteristics of the samples. Therefore, $\mu$ XRD can be widely used in studying lunar and other extraterrestrial samples as a simple, intuitive, and effective experimental method.

Furthermore, the crystalline state of particles in lunar samples can provide mineralogical evidence for identifying significant lunar surface geological processes. The appearance of glassy material suggests amorphization owing to high-energy thermal motion. The complex coexistence of crystals and glass in this study is attributable to the impact events of meteorites on the Moon surface.

\section{AUTHOR INFORMATION}

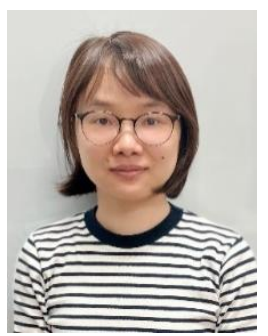

Lingya Ma is an associate professor at the Guangzhou Institute of Geochemistry, Chinese Academy of Sciences (GIGCAS) in Guangzhou, China. She is a member of the Youth Innovation Promotion Association, CAS. She completed her $\mathrm{Ph} . \mathrm{D}$. in mineralogy from the GIGCAS in 2016. The areas of her interest are using state-ofthe-art instrumentation to study the structure evolution and surface reactivity of phyllosilicate minerals, and the role of clay minerals on the formation of regolith-hosted rare earth elements deposits. She is also the manager of the micro-XRD lab in the GIGCAS. Emphasis is placed upon understanding the structure of minerals at the micro and nano level so that analysis results can be reliably used to clarify geological processes.

\section{Corresponding Author}

*L.Y. Ma

Email address: malingya@gig.ac.cn

Notes

The authors declare no competing financial interest.

\section{ACKNOWLEDGMENTS}

The Chang'e-5 lunar samples were allocated by the China National Space Administration. This work was financially supported by the National Natural Science Foundation of China (41921003, 42172049, and 41825003); the Science and Technology Planning Project of Guangdong Province, China (2017GC010128 and 2020B1212060055); and the Youth Innovation Promotion Association CAS (2018387).

\section{REFERENCES}

1. J. J. Papike, G. Ryder, and C. K. Shearer, in Planetary Materials, Reviews in Mineralogy, Vol. 36, 5-001-5-234, Mineralogical Society of America, 1998.

2. G. J. Taylor, M. J. Drake, M. E. Hallam, U. B. Marvin, and J. A. Wood, Proceedings of the Lunar Science Conference, 1973, 1, 553-568. https://ntrs.nasa.gov/search.jsp?R=19740040094

3. X. J. Zeng, X. Y. Li, J. Z. Liu, B. Mo, W. Yu, and H. Tang, J. Geophys. Res-Planet., 2020, 12, 1-15. https://doi.org/10.1029/2020JE006674

4. J. W. Delano, J. Geophys. Res-Sol. Ea., 1986, 91, 201-213. https://doi.org/10.1029/jb091ib04p0d201

5. S. K. Noble, L. P. Keller, and C. M. Pieters, Meteori. Planet. Sci., 2005, 3, 397-408. https://doi.org/10.1111/j.1945-5100.2005.tb00390.x

6. G. J. Taylor, K. Keil, and R. D. Warner, J. Theor. Biol., 1977, 1, 9-13. https://doi.org/10.1016/0022-5193(82)90385-X 
7. D. T. Vaniman, S. F. Lellis, J. J. Papike, and K. L. Cameron, Proceedings of the Seventh Lunar Science Conference, 1976, 199-239. https://ntrs.nasa.gov/citations/19740040094

8. S. B. Simon, J. J. Papike, and D. T. Vaniman, Proceedings of the Ninth Lunar Science Conference, 1978, 9, 1813-1826. https://www.researchgate.net/publication/23603767_The_Apollo_1 6_drive_tube_6000960010_I_-_Modal_petrology

9. J. L. Warner, C. H. Simonds, and W. C. Phinney, Proceedings of the Fourth Lunar Science Conference, 1973, 1, 481-504. https://ntrs.nasa.gov/citations/19740040090

10. K. J. Houck, J. Geophys. Res.-Sol. Ea., 1982, 87, A197-A209. https://doi.org/10.1029/JB087iS01p0A197

11. R. A. Zeigler, R. L. Korotev, B. L. Jolliff, L. A. Haskin, and C. Floss, Geochim. Cosmochim. Acta, 2006, 24, 6050-6067. https://doi.org/10.1016/j.gca.2006.08.040

12. K. J. Houck, J. Geophys. Res.-Sol. Ea., 1982, 87, A210-A220. https://doi.org/10.1029/JB087iS01p0A210

13. G. J. Taylor, B. R. Hawke, and P. D. Spudis, Workshop on Moon Beyond, 2002, 3049. https://www.lpi.usra.edu/meetings/moon2002/pdf/3049.pdf

14. G. J. Taylor, L. Martel, P. G. Lucey, J. Jeffrey, J. J. Gillis-Davis, F. B. David, and S. Philippe, Geochim. Cosmochim. Acta, 2019, 266, 17-28. https://doi.org/10.1016/j.gca.2019.07.046

15. J. B. Adams and T. B. McCord, Science, 1971, 171, 567-571. https://doi.org/10.1126/science.171.3971.567

16. W. Cassidy and B. W. Hapke, Icarus, 1975, 25, 371-383. https://doi.org/10.1016/0019-1035(75)90002-0

17. B. W. Hapke, W. Cassidy, and E. Wells, The Moon, 1975, 13, 339-353. https://doi.org/10.1007/BF00567525

18. P. M. Bell, H. K. Mao, and R. A. Weeks, Proceedings of the Seventh Lunar Science Conference, 1976, 2543-2559. https://ui.adsabs.harvard.edu/abs/1976LPSC....7.2543B/abstract

19. M. R. M. Izawa, R. L. Flemming, N. R. Banerjee, and P. J. A. McCausland, Meteori. Planet. Sci., 2011, 5, 638-651. https://doi.org/10.1111/j.1945-5100.2011.01180.x

20. B. Ralph, Mater. Charact., 2009, 1, 134. https://doi.org/10.1016/j.matchar.2009.08.002

21. R. L. Flemming, K. A. Salzsauler, B. L. Sherriff, and N. V. Sidenko, Can. Mineral., 2005, 43, 1243-1254. https://doi.org/10.2113/gscanmin.43.4.1243

22. R. L. Flemming, Can. J. Earth Sci., 2007, 9, 1333-1346. https://doi.org/10.1139/E07-020

23. L. A. Taylor, A. Patchen, D. H. S. Taylor, J. G. Chambers, and D. S. McKay, Icarus, 1996, 124, 500-512. https://doi.org/10.1006/icar.1996.0226

24. C. Kittel and R. W. Hellwarth, Phys. Today, 1957, 10, 43-44. https://10.1063/1.3060399

25. L. V. Azároff, M. J. Buerger, and M. M. Fishman, J. Electrochem. Soc., 1959, 106, 15C. https://doi.org/10.1149/1.2427251

26. W. A. Paciorek, M. Meyer, and G. Chapuis, Acta Crystallogr. A, 1999, A55, 543-557. https://doi.org/10.1107/S0108767398015037

27. S. Hu, H. He, J. L. Ji, Y. T. Lin, H. J. Hui, M. Anand, R. Tartèse, Y. H. Yan, J. L. Hao, R. Y. Li, L. X. Gu, Q. Guo, H. Y. He, and Z. Y. Ouyang, Nature, 2021, 600, 49-53. https://doi.org/10.1038/s41586-021-04107-9

28. J. Epp, Materials Characterization Using Nondestructive Evaluation (NDE) Methods, 81-124, Woodhead Publishing, 2016. https://doi.org/10.1016/B978-0-08-100040-3.00004-3
29. J. Kawai, C. Suzuki, H. Adachi, T. Konishi, and Y. Gohshi, Phys. Rev. B, 1994, 50, 11347-11354. https://doi.org/10.1103/PhysRevB.50.11347

30. B. W. Hapke, Ann. Ny. Acad. Sci., 1965, 123, 711-721. https://doi.org/10.1111/j.1749-6632.1965.tb20395.x

31. L. P. Keller and D. S. McKay, Science, 1993, 261, 1305-1307. https://doi.org/10.1126/science.261.5126.1305

32. S. J. Wentworthi, L. P. Keller, D. S. Mckay, and R. V. Morris, Meteor. Planet. Sci., 1999, 34, 593-603. https://doi.org/10.1111/j.1945-5100.1999.tb01366.x

33. R. Christoffersen, D. S. McKay, and L. P. Keller, Meteor. Planet. Sci., 1996, 31, 835-848. https://doi.org/10.1111/j.1945-5100.1996.tb02117.x

34. T. Noguchi, M. Kimura, T. Hashimoto, M. Konno, T. Nakamura, M. E. Zolensky, R. O. Kazaki, M. Tanaka, A. Tsuchiyama, A. Nakato, T. Ogami, H. Ishida, R. Sagae, S. Tsujimoto, T. Matsumoto, J. Matsuno, A. Fujimura, M. Abe, T. Yada, T. Mukai, M. Ueno, T. Okada, K. Shirai, and Y. Ishibashi, Meteor. Planet. Sci., 2014, 49, 188-214. https://doi.org/10.1111/maps.12111

35. T. Noguchi, T. Nakamura, M. Kimura, M. E. Zolensky, M. Tanaka, T. Hashimoto, M. Konno, A. Nakato, T. Ogami, A. Fujimura, M. Abe, T. Yada, T. Mukai, M. Ueno, T. Okada, K. Shirai, Y. Ishibashi, and R. Okazaki, Science, 2011, 333, 1121-1125. https://dx.doi.org/10.1126/science.1207794

36. T. Matsumoto, A. Tsuchiyama, A. Miyake, T. Noguchi, M. Nakamura, K. Uesugi, A. Takeuchi, Y. Suzuki, and T. Nakano, Icarus, 2015, 257, 230-238. https://dx.doi.org/10.1016/j.icarus.2015.05.001

37. L. V. Moroz, A. T. Basilevsky, T. Hiroi, S. S. Rout, D. Baither, C. H. Van-Der-Bogert, O. I. Yakovlev, A. V. Fisenko, L. F. Semjonova, V. S. Rusakov, D. A. Khramov, N. G. Zinovieva, G. Arnold, and C. M. Pieters, Icarus, 2009, 202, 336-353. https://doi.org/10.1016/j.icarus.2009.02.007

38. B. W. Hapke, Planet. Space Sci., 1968, 16, 101-110. https://doi.org/10.1016/0032-0633(68)90047-0

39. K. Wang, F. Moynier, F. A. Podosek, and J. Foriel, Earth Planet. Sci. Lett., 2012, 337-338, 17-24. https://doi.org/10.1016/j.epsl.2012.05.021

40. R. L. Baron, E. Bilson, T. Gold, R. J. Colton, B. W. Hapke, and M. A. Steggert, Earth Planet. Sci. Lett., 1977, 37, 263-272. https://doi.org/10.1016/0012-821X(77)90172-8

41. T. Gold, E. Bilson, and R. L. Baron, Philosophical Transactions of the Royal Society of London Series A, 1977, 285, 427-431. https://ntrs.nasa.gov/citations/19770046369

42. L. X. Gu, B. Zhang, S. Hu, T. Noguchi, H. Hidaka, and Y. T. Lin, Icarus, 2017, 303, 47-52. https://doi.org/10.1016/j.icarus.2017.12.028

43. C. M. Pieters, E. M. Fischer, O. Rode, and A. Basu, J. Geophys. Res-Planet., 1993, 98, 20817-20824. https://doi.org/10.1029/93JE02467

44. P. H. Warren and G. W. Kallemeyn, Proceedings of the NIPR Symposium on Antarctic Meteorites, 1991, 4, 91-117. https://adsabs.harvard.edu/full/1991AMR.........91W

45. R. L. Korotev, R. A. Zeigler, and B. L. Jolliff, Geochim. Cosmochim. Acta, 2003, 67, 4895-4923. https://doi.org/10.1016/j.gca.2003.08.001

46. A. Greshake, R. T. Schmitt, D. Stoffler, M. Pätsch, L. Schultz, Meteor. Planet. Sci., 2001, 36, 459-470. https://doi.org/10.1111/j.1945-5100.2001.tb01886.x 
47. J. Gross, and A. H. Treiman, J. Geophys. Res-Planet., 2011, 116, 1-9. https://doi.org/10.1029/2011JE003858

48. T. J. Bematowicz, R. H. Nichols, and C. M. Hohenberg, Lunar and Planetary Science Conference, 1994, 25, 105. https://ui.adsabs.harvard.edu/abs/1994LPI....25..105B/abstract

49. B. M. French, Technical Report: Lunar and Planetary Institute, 1998, 80, 31-61.

50. D. Stöfler, K. Keil, and E. R. D. Scott, Geochim. Cosmochim. Acta, 1991, 55, 3845-3867. https://doi.org/10.1016/0016-7037(91)90078-J

51. L. W. Snee and T. J. Ahrens, Proceedings of the Lunar Science Conference, 1975, 6, 833-842. https://ntrs.nasa.gov/citations/19780062739
52. Y. Z. Yang, H. Zhang, Z. W. Wang, Y. Yuan, S. L. Li, W. Hsu, and C. J. Liu, Astrono. Astrophys., 2017, 597, A50. https://doi.org/10.1051/0004-6361/201629327

53. J. Dran, L. Durrieu, C. Jouret, and M. Maurette, Earth. Planet. Sci. Lett., 1970, 9, 391-400. https://doi.org/10.1016/0012-821X(70)90003-8

54. F. Hörz, and W. L. Quaide, The Moon, 1973, 6, 45-86. https://doi.org/10.1007/BF02630652

55. J. C. M. Butil, M. L. F. Magsisi, J. H. Pua, P. K. Se, and R. Sagum, Int. J. Comput. Commun., 1978, 3, 199-202. https://doi.org/10.7763/IJCCE.2014.V3.319

56. J. W. Delano and K. Livi, Geochim. Cosmochim. Ac., 1981, 11, 2137-2149.

https://doi.org/10.1016/0016-7037(81)90066-1 